\title{
La gestión de cobranza y la automatización por una base de datos.
}

The collections process and automation for a database.

Velasteguí López Efraín. ${ }^{1}$, Sánchez Intriago Carolina. ${ }^{2}$

DOI: https://doi.org/10.33262/visionariodigital.v1i3.257

\section{Resumen.}

El objetivo de la investigación es contribuir a la importancia de la recaudación de deudas de los clientes de la Cooperativa de Ahorro y Crédito Innovación Andina en el área de cobranza. Se ha orientado el desarrollo de la solución debido a los altos niveles de morosidad presentados y los altos valores de deuda de diversos clientes contraídos con la cooperativa en el sector financiero.

En la actualidad la Cooperativa de Ahorro y Crédito, realiza el registro y control de las actividades llevadas a cabo durante el proceso de recuperación de una deuda, en general de manera manual, lo cual genera en muchos casos perdida de información, desactualización del estado de la cartera y retrasó en la realización de actividades. Debido a esto, el resultado de los créditos brindados no es siempre exitoso ya que finalmente no termina recuperándose lo esperado en el tiempo acordado y se genera un desbalance en las cuantas.

Es así, como durante la presente investigación, se realizó el análisis, de la importancia de una base de Datos para el sistema de recaudación de forma provechosa. Logrando gestionar de forma más eficiente los recursos para atacar cada uno de los casos de la mejor manera posible y brindar soporte a las actividades llevadas a cabo a lo largo del proceso alcanzado un control sobre cada uno de los clientes deudores. Esto permitirá respaldarla las decisiones tomadas durante el proceso para obtener el resultado esperado que es la recuperación de la deuda.

\footnotetext{
${ }^{1}$ Universidad Técnica de Ambato, Ambato, Ecuador, le.velastegui@uta.edu.ec

2 Universidad técnica de Cotopaxi. Cotopaxi ecuador, sanchezs@gmail.com
} 
Palabras claves: Gestión de cobranza, automatización por una base de datos.

\section{Introducción.}

En la actualidad, la Cooperativa de Ahorro y Crédito Innovación Andina del Cantón la Maná dedicada a prestar servicios de créditos financieros, realiza el registro y control de actividades llevadas a cabo durante el proceso de recuperación de una deuda, en general, de manera manual, lo cual genera en muchos casos perdidas de información, desactualización del estado de la carteras y retraso en la realización de actividades. Debido a esto, el resultado de los créditos brindados no es siempre exitoso ya que finalmente no se recupera en el tiempo acordado y se genera un desbalance en las cuentas que puede llevar a serios problemas al asesor de cobranza.

Es así que mediante el presente ensayo, se conocerán las necesidades de la cooperativa en el área de cobranza y se seguirá lineamientos dados por métodos y procedimientos adecuados, cuya información permita obtener un sistema que administre la cartera de clientes deudores de la Cooperativa de Ahorro y Crédito de forma provechosa. Logrando gestionar de forma más eficiente los recursos para atacar cada uno de los casos de la mejor manera posible y brindar soporte a las actividades llevadas a cabo a lo largo del proceso alcanzado un control sobre cada una de las comunicaciones dadas con el deudor. Esto permitirá respaldar las decisiones tomadas durante el proceso para obtener el resultado esperado que es la recuperación de la deuda.

Las Bases de Datos juegan un papel importante en la mayoría de las áreas donde se utilizan computadores, permitiendo almacenar grandes volúmenes de datos acerca de la empresa, los cuales son percibidos a través de los usuarios, de la misma manera la información obtenida de los datos almacenados debe estar en una forma que sirva para administrar, planear, controlar y tomar decisiones dentro de una organización.

\section{Desarrollo.}

\section{Que es una base de datos.}

Un base de datos según (Kroenke, 2003; Date, 2001 \& Cisnero, 1998) afirman que es un sistema que se establece alrededor de un archivo de datos compartidos en forma centralizada, integrada y optimizada, que subraya la independencia de los programas y de los datos. En otras palabras, un sistema de base de datos es el recipiente donde es almacenada la informacion, un conjunto de datos persistentes que es utilizado por el sistema de aplicación de alguna empresa dada. El termino base de dato tiene mucha interpretacion diferente, se ha usado para referirse a un conjunto de tarjetas indexadas a los volumenes de datos que un gobierno recopila acerca de sus ciudadanos se puede decir que tiene caracteristicas de ser: compartido, integrado y proporciona independencia de datos y de programas.

Mediante mi criterio propio se podría decir que una base de datos permite obtener acceso a una información actualizada y precisa, tener un diseño correcto para lograr sus objetivos 
de trabajar con una base de datos, tiene sentido invertir el tiempo necesario para obtener información sobre los principios de un buen diseño, es muy probable que al final se obtenga una base de datos que se ajuste a sus necesidades y que puede adoptarse fácilmente al cambio, lo que se espera es una base de datos que nos permita guardar grandes cantidades de información de forma organizada para que luego podamos encontrar y utilizar fácilmente, se puede decir que una base de datos se compone de una o más tablas que guardan un conjunto de datos. Cada tabla tiene una o más columnas y filas, las columnas guardan una parte de la información sobre cada elemento que queramos guardar en la tabla, cada fila de la tabla conforma un registro.

Los diseñadores de base de datos generalmente organizan los datos para modelar aspectos de la realidad de una manera que respalda los procesos que requieren información, como modelar la disponibilidad de habitaciones en hoteles de una manera que permita encontrar un hotel con vacantes. El sistema de administración de base de datos es el software que interactúa con los usuarios finales, las aplicaciones y la propia base de datos para capturar y analizar datos. Un DBMS de propósito general permite la definición, creación, consulta, actualización y administración de base de datos. Una base de datos generalmente se almacena en un formato especifico de DBMS que no es portátil, pero diferentes DBMS pueden compartir datos mediante el uso de estándares tales como SQL y ODBC o JDBC. La suma total de la base de datos, el DBMS y sus aplicaciones asociadas puede dominarse "sistema de base de datos". A menudo, el término "base de datos" se usa para transferir a cualquiera de los DBMS, el sistema de base de datos a una aplicación asociada con base de datos.

Anteriormente se utilizaba el sistema de procesamiento de archivos en el cual toda la información de la empresa se almacenaba en archivos permanentes manejados por un sistema operativo convencional, se tenía que escribir diversos programas de aplicación para extraer registros y añadir registros a los archivos adecuados.

\section{Importancia de una Base de Datos.}

Según (Kroenke, 2003; Benítez, 2017 \& Acid, 2005) afirman que en la actualidad la recopilación de datos es fundamental para que una empresa o institución mantenga sus relaciones. Por este motivo se le brinda una gran importancia al mantenimiento de la base de datos y también al constante crecimiento de la misma. Es importante que la base de datos contenga ciertos datos fundamentales de la persona, como por ejemplo su teléfono, dirección de correo electrónico y la dirección postal. Estas bases de datos son dinámicas, pues se modifican todo el tiempo a diferencia de las estáticas que suelen recopilar información o documentos históricos. Las bases de datos se originaron desde mitad de los años sesenta, pero en 1970, se propuso un modelo relacional de datos para grandes bancos de datos compartidos y hasta el momento este ha sido el modelo que se ha mantenido. A muchos de nosotros y organizaciones utilizamos las bases de datos, como método de encontrar información de forma rápida y con menos riesgo de pérdidas. Entre 
más datos sean almacenados en una base de datos se convierte más útil; esta puede llegar a proporcionar a las personas el acceso de datos, donde podemos visualizar ingresar o actualizar información.

De mi punto de vista nuestro día a día las bases de datos juegan un papel mucho más importante de lo que pensamos. Podemos pasar por diferentes establecimientos y negocios, sin pararnos a pensar que si no fuera por las base de datos, sería muy complicado realizar tareas tan sencillas como comprobar si el pantalón que buscamos en nuestras tiendas de ropa habitual está disponible en nuestra talla. Las bases de datos constituyen una parte fundamental de los sistemas de información en las que están integrados.

El estado actual de la tecnología de base de datos en el mundo, es el resultado de la revolución que a lo largo de décadas ha tenido lugar en el procesamiento de los datos y en la gestión de información. Esta tecnología se ha ido desarrollando a lo largo del tiempo desde los métodos más primitivos de los años cincuenta, hasta los potentes sistemas de hoy en día, empujada por un lado por la demanda y las necesidades de la gestión de la información y restringida por las limitaciones de la tecnología.

Pero desde ya hace décadas que se establecieron modelos para su almacenamiento y gestión, aparecieron los primeros modelos. Los datos han adoptado una importancia de grandes dimensiones en la actualidad, más si cabe, de ahí la relevancia de los modelos de base de datos. Siempre han tenido un papel clave en la informática, en la empresa y en muchos sectores, ya que la recopilación y gestión de los datos son fundamentales para que las empresas o instituciones mantengan su orden y sus relaciones. Por todo esto, es importante establecer efectivos modelos de gestión de base de datos y un concreto mantenimiento y mejora.

\section{Sistema de Gestión de Base de Datos.}

Según (MAD-Eduforma, 2003; Cobo, 2000 \& Gómez, 1998) nos dan a conocer que sistema de gestión de base de datos es el conjunto de herramientas que ayudan al usuario a gestionar información almacenada en una base de datos, se puede modificar, introducir o eliminar información. Los SGBD surgieron para paliar las desventajas del uso de un sistema de procesamiento de archivos tradicionales, se idearon para solucionar estos problemas. Intentan conseguir la integración de ficheros de datos, de sus estructuras y de las aplicaciones que las manejan, así como de muchos aspectos adicionales de seguridad y control, salvaguardia, accesos simultáneos, etc. Un sistema de gestión de base de datos es un software o conjunto de programas que permite crear y mantener una base de datos. El SGBD actúa como interfaz entre los programas de aplicación (Usuarios) y el sistema operativo. El objetivo principal de un SGBD es proporcionar un entorno eficiente a la hora de almacenar y recuperar la información de la base de datos. 
Se puede entender que Un Sistema Gestor de Base de Datos es un conjunto de programas que no podemos ver, pero estos son los que administran y gestionan la información que contiene una base de datos. Gracias a este sistema de software invisible para el usuario final, compuesto por un lenguaje de definición de datos, un lenguaje de manipulación y consulta, es posible gestionar los datos a distintos niveles. Tanto almacenar, modificar y acceder a la información como realizar consultas y hacer análisis para generar informes. También se puede entender que un Sistema de Gestión de Base de datos es como una colección de datos relacionados entre sí, estructurados y organizados dentro del ecosistema conformado por ese conjunto de programas que acceden a ellos y facilitan su gestión. Un gestor controla cualquier operación ejecutada por el usuario contra la base de datos. Para ello, se utilizan herramientas específicas, como sistemas de búsqueda y de generación de informes, además de distintas aplicaciones.

Los modelos de datos definen con claridad cómo se modela la estructura lógica de una base de datos. Estos, son entidades necesarias para introducir la abstracción en un SGBD, entendiendo por abstracción al proceso de aislar un elemento de su contexto o del resto de elementos que lo pueden acompañar. Un modelo de base de datos incluye, además, las relaciones y limitaciones que determinan como se pueden almacenar los datos y acceder a ellos. Actualmente, vivimos en la era de la tecnología y los datos, ya que estos tienen infinidad de usos: económicos, sociales, etc. Se han convertido en foco de mayor estudio e inversión.

También se dice que permite la definición de base de datos a distintos niveles de abstracción, así como su manipulación, garantizando tanto la seguridad como la integridad y consistencia de los mismos. Siempre de forma separada a los programas o aplicaciones que los gestionan, sin tener que modificar estos. Muchas de las funciones de un gestor de base de datos vienen contenidas en la breve definición realizada. Como hemos apuntado, un SGBD nos permite definir los datos, asi como manipular, aplicar medidas de seguridad e integridad y recuperarlos / restaurarlos tras un posible fallo.

\section{Tipos de Base de Datos.}

Hay bases de datos relacionales, como MySQL, SQL Server y Oracle. Como su nombre lo indica utilizan el modelo relacional y siempre es mejor usarlas cuando los datos son consistentes y ya tienen algo planificado. Aunque estas bases de datos relacionales proporcionan una buena estructura y accesibilidad para la mayoría de los datos, también tienen limitaciones que han dado lugar a unos nuevos tipos de base de datos que abordan necesidades más específicas para tratar con recursos de datos extremadamente grandes y complejos.

\section{¿Qué es MySQL?.}

Según (Thibaud, 2006; Cobo, 2005 \& Arias 2014) aseguran que MySQL es un sistema de administración de base de datos relacionales rápido, sólido y flexible. Es ideal para 
crear bases de datos con acceso desde páginas web dinámicas, para la creación de sistemas de transacciones ol-line o para cualquier otra solución profesional que implique almacenar datos, teniendo la posibilidad de realizar múltiples y rápidas consultas. MySQL dispone de dos programas principales: el servidor o motor y el cliente o motor MySQL. El primero de ellos se encarga de estar a la espera de posibles peticiones recibidas de los clientes; mientras que el segundo es el encargado de actuar de interfaz con el usuario. Para comenzar a utilizar el sistema MySQL lo primero que se debe hacer es arrancar su programa servidor. Este programa se ejecutara en un segundo plano como un proceso o servicio, no tiene una interfaz de usuario y su único propósito es estar a la espera de que alguien se conecte a él y le envié una solicitud.

Podría afirmar mediante los conceptos que anteriormente afirman los autores que MySQL es un sistema de gestión de datos relacional de código abierto, basado en lenguaje de consulta estructurado. A pesar de que se puede utilizar en una amplia gama de aplicaciones, MySQL se asocia más con las aplicaciones basadas en la web y la publicación en línea y es un componente importante de una pila empresarial. Este gestor de base de datos en multihilo y multiusuario, lo que permite ser utilizado por varias personas al mismo tiempo, e incluso, realizar varias consultas a la vez, lo que lo hace nuevamente versátil.

\section{¿Qué es SQL Server?}

Según (Dooley, 2000; Delgado, 1997 \& England, 1999) nos afirman que SQL Server es un sistema de gestión de base de datos relacionales de Microsoft que esta diseñado para el retorno empresarial. SQL Server se ejecuta en T-SQL (Transact-SQL), un conjunto de extensiones de programación de Sybase y Microsft que añaden varias características a SQL estándar, incluyendo control de transacciones, excepción y manejo de errores, procesamiento fila, así como variables declaradas.

Se puede decir que es un sistema de gestión de base de datos el cual da soporte de transacciones, soporta procesamiento almacenados se dice también que este sistema incluye una versión reducida, llamada MSDE con el mismo motor de base de datos pero orientado a proyectos más pequeños, que en su versión 2005 pasa a ser SQL se distribuye en forma gratuita.

\section{Oracle.}

Según (Orfali, 2017; Chaparro, 2012 \& Duran, 2007) aseguran que Oracle es básicamente una herramienta cliente/ servidor para la gestión de base de datos. Es un producto vendido a nivel mundial, aunque la gran potencia que tiene y su elevado precio hacen que solo se vea en empresas muy grandes y multinacionales, por norma general. En el desarrollo de páginas web pasa lo mismo: como es un sistema muy caro no esta tan 
extendido como otras bases de datos, por ejemplo, Access, MySQL, SQL Server, etc. Oracle se basa en la tecnología, pues bien para su utilización primero sería necesario la instalación de la herramienta servidor (Oracle 8i) y posteriormente podríamos atacar a la base de datos desde otros equipos con herramientas de desarrollo como Oracle Designer y Oracle Developer, que son las herramientas básicas de programación. Para desarrollar en Oracle utilizamos PL/SQL un lenguaje de quinta generación, bastante potente para tratar y gestionar la base de datos, también norma general se suele utilizar SQL al crear un formulario.

Se podría decir que Oracle es un sistema de gestión de base de datos relacional Ha sido criticada por algunos especialistas la seguridad de la plataforma, y las políticas de suministro de parches de seguridad, modificadas a comienzos de 2005 y que incrementan el nivel de exposición de los usuarios. En los parches de actualización provistos durante el primer semestre de 2005 fueron corregidas 22 vulnerabilidades públicamente conocidas, algunas de ellas con una antigüedad de más de 2 años.

Oracle es básicamente un herramienta cliente/servidor para la gestión de base de datos, es un producto vendido a nivel mundial, aunque la gran potencia que tiene y su elevado precio hace que solo se vea en empresas muy grandes y multinacionales, por norma general. En el desarrollo de páginas Web pasa lo mismo como es un sistema muy caro no está tan extendido como otras bases de datos, por ejemplo, Access, MySQL, SQL Server etc. Oracle como antes lo mencionamos se basa en la tecnología cliente/ servidor, pues bien, para su utilización primero sería necesario la instalación de la herramienta servidor ( Oracle8i ) y posteriormente podríamos atacar a la base de datos desde otros equipos con herramientas de desarrollo como Oracle Designer y Oracle Developer, que son las herramientas de programación sobre Oracle a partir de esta premisa vamos a desarrollar las principales acepciones de Oracle y sus aplicaciones en las distintas ares de trabajo.

\section{Comparación de My SQL, SQL Server y Oracle.}

Se analizó algunos sistemas gestores de base de datos existentes, tratamos de darle una idea a grandes rasgos de lo que era cada uno y los beneficios que ofrecen para que al momento de necesitar de estas herramientas se pudiera elegir la que mejor se acomode a las necesidades, concentrando la investigación en los 3 principales gestores de la actualidad que es Oracle, MySQL Y SQL Server.

Es muy importante una buena elección de un sistema gestor de base de datos. En mi criterio personal escogería My SQL ya que si se desarrolla aplicaciones de mediano/ pequeño y se utiliza PHP la mejor opción sería My SQL. Pero si se piensa en un desarrollo empresarial, resistentes y de gran escala, SQL Server debería ser el aliado perfecto.

Gráfico N 1: Uso de las bases de datos más utilizadas en los últimos años 


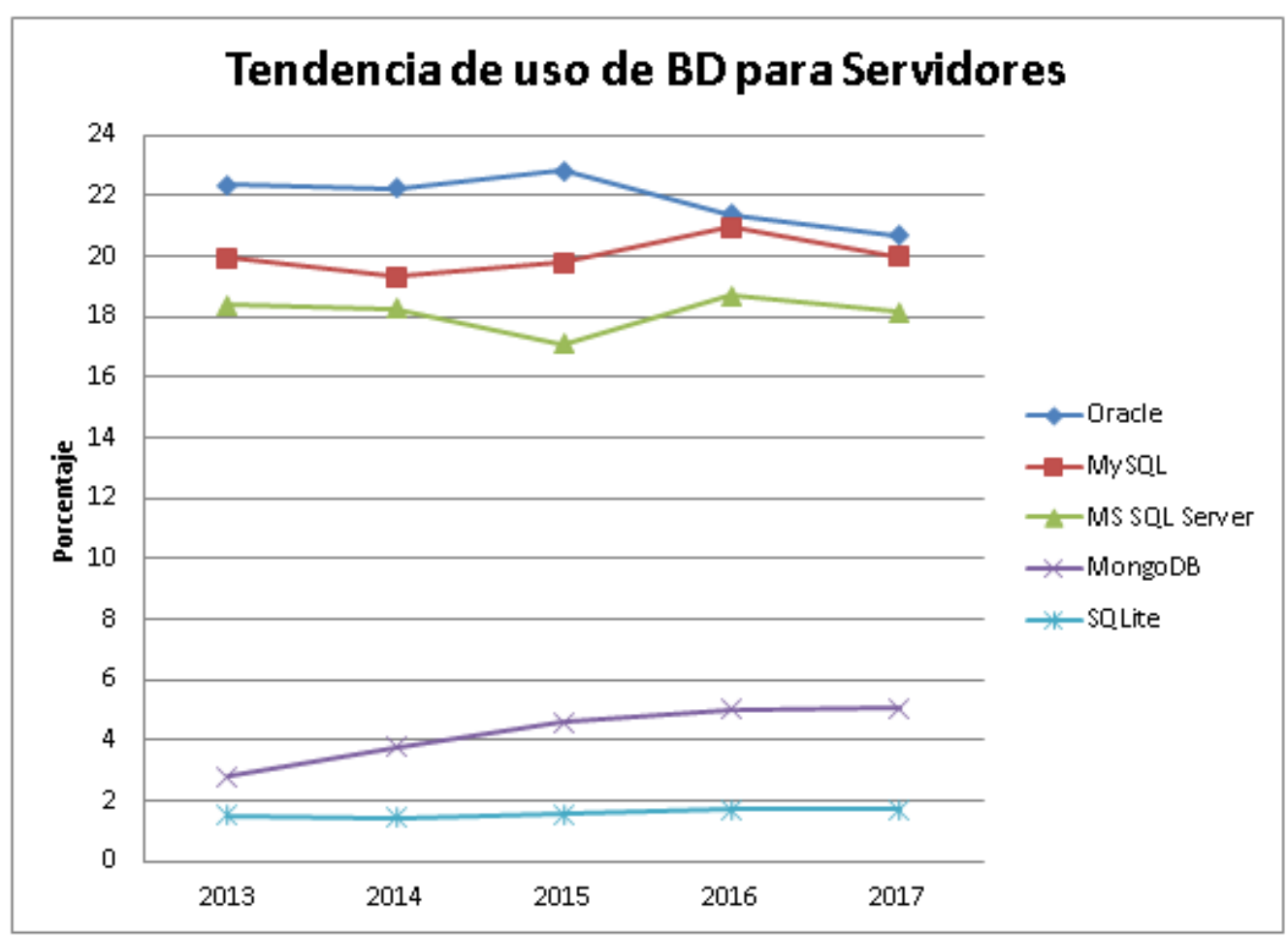

Fuente: http://diariecillodetareas.blogspot.com/p/bases-de-datos-mas-utilizadas-enlas.html

Autor: Propias del Autor

\section{El Proceso de la cobranza.}

Según (Morales, 2014; Molina, 2002 \& González, 2002) certifican que es un proceso complejo en el cual deben tomar muchas consideraciones puesto que los pasos a seguir no siempre son los mismos en base a la deuda especifica que se está tratando. Debido a la época de crisis en que actualmente nuestro entorno económico está sujeto, hace que las personas adquieran pasivos con una gran facilidad, esto mismo hacen que caigan en una mora de sus pagos en los créditos contraídos. En la actualidad los diversos métodos de cobranza han opacado el "Proceso de cobranza" que anteriormente se tenía, ya que en algunas organizaciones han adoptado técnicas poco favorables para acelerar los cobros y controlar en forma estrecha los pagos, este proceso hace que algunos agentes de cobranza realicen la gestión con maltrato, ofensas y amenazas.

En mi punto de vista el sistema de cobranza es el proceso mediante el cual se hace efectiva la percepción de un pago en concepto de una compra, de la prestación de un servicio, de la cancelación de una deuda, etc. Esta puede ser emprendida por la misma empresa que debe recibir el pago de un área dedicada especialmente a esta carencia, o puede encontrarse a otra institución. Una persona dominado cobrador se hace presente en los domicilios identificados para el abono del monto estipulado. Manejar las finanzas no es una terea sencilla en una empresa, que deberían ser proactivas y mantener el orden para lograr el éxito en su ejecución. Expertos brindan sus claves para gestionar mejor el proceso de cobro. 
El sistema de cobranza, conocido también como factores, es una alternativa de financiamiento que se delega a una empresa especializada, y que en ocasiones puede ser una gran alternativa para responder a las necesidades financieras de las PYMES. El sistema de cobranza consiste en entregar a la empresa contratada los cobros de los clientes de las empresas contratantes, con el fin de que gestione el cobro con los mecanismos que se consideren convenientes, bajo las reglas y disposiciones del ente especializado. Dicho modelo permite mayor eficiencia y certidumbre en cuestiones operacionales al ser gestionados por un ente especializado.

Tabla N 1: Ventajas y desventajas del proceso de cobranza.

\begin{tabular}{|c|c|}
\hline Ventajas & Desventajas \\
\hline $\begin{array}{l}\text { Asegura la viabilidad de recuperación de la deuda } \\
\text { Otorga liquidez a la empresa (porque no tiene que } \\
\text { esperarse al cobre efectivo de los créditos). } \\
\text { Es una forma de financiamiento flexible y } \\
\text { accesible. } \\
\text { Otorga una sistematización de la información de } \\
\text { los deudores, y con ello es posible conocerlos y } \\
\text { tomar mejores decisiones en torno a ellos. } \\
\text { La empresa de fáctoreo tiene personas calificadas } \\
\text { para resolver los problemas de cobro, y puedan } \\
\text { servir de mediadores entre partes. } \\
\text { La empresa contratando reduce costos de } \\
\text { operación, e incluso puede significar eliminar por } \\
\text { completo el departamento de cobros. } \\
\text { Es un sistema de financiamiento que crece con las } \\
\text { ventas, lo que conlleva a mejor capacidad para la } \\
\text { atención de la demanda que va creciendo. } \\
\text { Predice los flujos de caja, pues al recibir con } \\
\text { certeza ciertos ingresos, es posible hacer una } \\
\text { planeación de flujo de caja. } \\
\text { Convierte a sus ventas a crédito en ventas de } \\
\text { contado. }\end{array}$ & $\begin{array}{l}\text { Para las empresas con crisis financiera temporal no } \\
\text { resulta de mucha ayuda. } \\
\text { Muchas empresas de cobranza estudian al cliente } \\
\text { (y su solvencia) y no a la empresa misma. } \\
\text { El cobrador solo se dejara las cuentas que le } \\
\text { convenga, no necesariamente las más difíciles. } \\
\text { Cuando son cobros pequeños, el fáctoreo aumenta, } \\
\text { los gastos administrativos, lo cual deviene en } \\
\text { inconveniente y resulta poco útil. } \\
\text { Si los plazos de las facturas son cortos, puede } \\
\text { aumentas el costo financiero, debido al porcentaje } \\
\text { de la comisión. }\end{array}$ \\
\hline
\end{tabular}

Fuente: Investigada.

Elaborado por: Carolina Sánchez. 


\section{Entrega y recepción de la cobranza}

Según (Ripia, 1976; Molina, 2005 \& Gitman, 2003) Nos afirman mediante sus referencias que se realiza la recepción de la cartera de cliente, con la cual ya se posee un acuerdo previo. Se debe definir las reglas de cobranza serán impuestas por la empresa pues en base a estas se realizan las siguientes actividades. Esta carta de deudores será el motivo de trabajo por parte del asesor de cobranza. Es un conjunto de operaciones necesarias para cancelar una obligación contraída. Esto implica una disminución de disponibilidades y por consiguiente también se producirá una disminución de deuda.

No se puede desconocer que el porcentaje de crédito en el mundo es cada vez mayor. Esto representa un mayor riesgo para las cooperativas debido a que el pago de los créditos no se ve reflejado en caja al momento de la entrega del producto o servicio. Por lo tanto, el proceso requiere ser más eficiente. No solo se trata de vender (entregar un producto o servicio), se requiere que haya un retorno de la inversión con los márgenes establecidos como metas y que además exista una relación comercial perdurable en el tiempo con los clientes.

\section{Clasificación de la deuda.}

Según (Millet, 2005; Banco Mundial, 2001 \& Ayala, 2004) nos afirman que es importante conocer quién es el cliente y cuál es su situación, así como cuáles fueron las condiciones dadas para otorgar el crédito. Se debe acceder a fuentes externas de información tales como centrales de riesgo, relaciones de deudores, etc. Luego, en base a este conocimiento del caso en particular se clasifica la deuda de acuerdo a criterios manejados por la empresa y que permitirán darle un mejor tratamiento tomando en consideración la naturaleza de la misma.

Se podría decir que cuando un banco, entidad, emite deuda ya sea por motivos de inversión o de financiación, en la relación contractual que se contrae, se corresponde con el deudor (emiten deuda y contraen la obligación de devolver el principal más intereses), y aquel que compra dicha deuda se convierte en su acreedor (son aquellos que desembolsan un principal y reciben unos intereses a cambio).

Siendo más precisos en la definición, la deuda es la obligación de devolver el capital en el plazo acordado y con el tipo de interés fijado. La cantidad adeudada es la suma del capital solicitado más los intereses que correspondan.

Cuando un banco, entidad, Estado...emite deuda ya sea por motivos de inversión o de financiación, en la relación contractual que se contrae, se corresponde con el deudor (emiten deuda y contraen la obligación de devolver el principal más intereses), y aquel que compra dicha deuda se convierte en su acreedor (son aquellos que desembolsan un principal y reciben unos intereses a cambio). 


\section{Gestión de la deuda.}

Según (Millet, Toussaint, \& Borresen, 2005) se refieren que la deuda es asignada a un gestor de cobranza de acuerdo a la clasificación que se le dio, de esta forma, se logró gestionar la forma óptica los recursos asignados casuísticas de mayor o menor dificultad a gestores con la suficiente experiencia, lo cual permite recuperar la cartera en cada uno de los casos y aprovechar al máximo los recursos que se posee. El gestor de cobranza será el encargado de comunicarse con el cliente por la vía correspondiente, notificarle la deuda y daré la posibilidad de realizar un financiamiento de la deuda si llegara a un acuerdo con el deudor y se diera el caso. También, se encarga de brindarle facilidades al deudor para que logre cancelar lo adeudado y poder reincorporarlo respetando siempre as reglas que caracterizan a esta cartera.

Se podría decir que gestión de la deuda es esencial en todas las entidades. Un buen trabajo en este campo pasará desapercibido, pero una mala labor puede llevar a la sociedad más sólida a la falta de liquidez y, finalmente, incluso a su desaparición. Podemos clasificar la deuda en exigible y no exigible, en función de la posibilidad del acreedor de reclamar el pago de la deuda. Dentro de la deuda no exigible incluimos las reservas, los resultados no distribuidos y el capital social de la empresa. La eficacia en la gestión de deudas impagadas adquiere un valor fundamental en la evolución de cualquier compañía, siendo esencial para la solvencia y para la propia supervivencia de las empresas en general, máxime si hablamos de empresas de tamaño reducido. Por ello, la profesionalización de esa gestión, así como la competencia en la misma, pueden marcar una significativa diferencia en los resultados de una empresa.

Expertos y habituados a gestiones de reclamación y negociación de deudas problemáticas, nos especializamos en gestión activa y presencial de GRANDES CUENTAS, con profesionalidad y adaptación a los requerimientos éticos, procedimentales y de calidad del cliente. Ofrecemos una experiencia de más de quince años en la reclamación y gestión de deudas impagadas con alta especialización y un servicio personalizado con soluciones a medida ("tailor-made") para nuestros clientes.

\section{Refinanciamiento de la deuda.}

Según (Reyno, Jhabvala, Ibanez, \& Martinez, 2000) se refieren que consiste en la modificación de un crédito existente, desembolsado por la institución crediticia, en respuesta a la incapacidad de pago de un cliente en el largo plazo. Generalmente, trae consigo ajustes en las condiciones iniciales del crédito y, también, puede incluir la ampliación del monto del mismo. Para que esto sea posible los clientes requieren cumplir algunas condiciones previas.

Lo habitual es que el refinanciamiento consista en una reducción de la cuota que se paga y en un recorte de la tasa de interés, permitiendo que el deudor pague el préstamo en un plazo más extenso. El objetivo es adaptar la devolución del crédito a los ingresos reales 
de quien debe saldar la deuda para que ésta no resulte impagable. Seguramente pensarás que conseguir la refinanciación de deudas con bancos es prácticamente imposible. Sin embargo, las entidades financieras, especialmente en épocas de crisis, no son reacias a la refinanciaciones, ya que generalmente les beneficia. Surge entonces la pregunta de cómo refinanciar una deuda con el banco. La respuesta es más sencilla de lo que parece, pues cualquier persona puede dirigirse al banco para solicitar una refinanciación, de forma similar a cuando se va a un banco para obtener un crédito. Los pasos son también parecidos, puesto que el banco ha de considerar el proyecto viable y además, los exigiera una serie de condiciones. Aun en el caso de los préstamos es frecuente que soliciten garantías, cuando se acude a solicitar una financiación es muy posible que el banco exija a más garantías.

Teniendo en cuenta todo lo anterior, es importante explicar en el banco de forma clara a la situación de la persona física o jurídica que solicita la refinanciación. También, que se tenga interés en hacerse cargo del préstamo y ser capaces de asumir los nuevos plazos negociados. Muchas veces, aunque la refinanciación implique pagar más dinero, puede constituir una interesante alternativa, especialmente cuando lo que se necesita es comprar tiempo. En este sistema de pago el exportador entrega a una entidad financiera documentos financieros (letras de cambio) y/o comerciales (facturas, listas de empaque, documentos de transporte, entre otros), con el fin de que dichos documentos lleguen a manos del comprador cuando el vendedor se lo indique a la entidad financiera correspondiente como contra pago o contra aceptación, es decir, previo pago y/o aceptación de una letra de cambio por parte del importador.

\section{Conclusiones.}

- Es necesario desarrollar un software capaz del registro y control del seguimiento realizado durante el proceso de recaudación de deudas y que además así se podría brindar accesibilidad al usuario y le va a permitir la adición de futuras funciones gracias a la escalabilidad del mismo. Esto debido a la elaboración de un prototipo al inicio de la etapa de implementación.

- Se debe implementar un método que asigna los gestores de cobranza con más experiencia al seguimiento de las deudas más difíciles de cobrar gracias a la construcción de un algoritmo que analice el comportamiento de los datos propios de los gestores y las deudas así como la información personal de los deudores. Se podrá demostrar el uso de este método para distribuir la carga de trabajo a los colaboradores ayudó a que todos tuviesen tareas acordes a sus capacidades y a aumentar la probabilidad de éxito al cobrar a cada una de las deudas.

- Se tiene que implementar una interface para realizar la carga masiva de la información de las carteras de deudas. Esta va a facilitar a los acreedores para que puedan transferir la información de las carteras para cuya cobranza contrataron a la empresa especializada. Esta funcionalidad brinda mucha transparencia en el envío de información y permite a los acreedores asegurarse de enviar toda la información necesaria para el cobro así como a la empresa de cobranza evitar la 
pérdida de información que se daba cuando esta tarea era efectuada de manera manual.

- Se deberá desarrollar distintos tipos de reporte, los cuales reflejan información valiosa a partir de los datos que almacena el sistema luego de que los usuarios registraron sus actividades en el mismo. Estos reportes brindaran un respaldo y apoyo constante a la toma de decisiones en las empresas de cobranza así como permiten conocer en tiempo real el avance en las carteras que vienen trabajando.

\section{Bibliografía.}

Acid Carrillo, S., Marin Ruiz, N., Medina Rodriguez, M., Pons Capote, O., \& Vila Miranda, A. (2005). Introduccion a las base de datos. Madrid: Copyright Internacional.

Arias, A. (2014). Bases de Datos con MySQL. Barcelona: IT Campus Academy.

Ayala Espino, J. (2001). Economia del sector público . Mexico: UNAM.

Banco Mundial. (2004). Estadísticas de la Deuda Externa: Guía para Compiladores y Usuarios. Washington: International Monetary Fund.

Benitez, M. A., \& Arias, A. (2017). Curso de Introduccion a la Administracion de Base de Datos. Madrid: IT Campus Academy.

Chaparro, M. (2012). Oracle 11 g PL/SQL: curso práctico de formación. Madrid: RC Libros.

Cisnero Gonzalez, J. L. (1998). Panorama sobre base de datos (un enfoque práctico). California: Autonoma de Baja California. II. t.

Cobo, A. (2000). Diseño y programacion de bases de datos. España: Editorial Visión Libros.

Cobo, A. (2005). PHP Y MySQL: Tecnología para el desarrollo de aplicaciones web. España: Ediciones Díaz de Santos.

Date, C. (2001). Introducción a los sistemas de base de datos (Sexta ed.). Mexico: Pearson Educación.

Delgado Garrón, A. (1997). Microsoft SQL Server 6.5: Guia Avanzada. Diseño y Administracion de Base de Datos Realacionales Cliente/ Servidor. Barcelona: Pretice Hall.

Dooley, S. (2000). SQL Server 7 Essential Reference. Barcelona: Sams Publishing.

Duran, L., Rodriguez, E., \& Santata, K. (2007). Base de datos con Visual Basic. Mexico: Marcombo. 
England, K., \& Stanley, N. (1999). The SQL Server 7.0 Hondbook: A Guide to Microsoft Database Computing. America: Digital Press.

Gitman, L. (2003). Principios de administracion financiera. Mexico: Person Educatión.

Gómez Gómez, A., \& De Abajo Martínez, N. (1998). Los sistemas de información en la empresa. Oviedo: Los sistemas de información en la empresa.

Gonzalez, E. R. (2002). La Negacion de los procesos de cobranza. Madrid: Editora Cosac Naify.

Kroenke, D. (2003). Procesamiento de base de datos. Mexico: Person Educación.

MAD-Eduforma. (2003). Cuerpo de Administrativos de Administracion General. Comunidad Autonoma de Madrid. Temario Volumen Ii. E-book. Madrid: MADEduforma.

Millet, D., Toussaint, E., \& Borresen, P. (2005). Deuda Banco Mundial. Quito: Editorial Damien Millet.

Molina Aznar, V. E. (2002). El gestor de Cobranza. mEXICO: Ediciones fiscales ISEF.

Molina Aznar, V. E. (2005). Estrategias de Cobranza en epoca de crisis. Mexico: Ediciones Fiscales ISEF.

Morales, A., Morales, J., \& Castro, A. (2014). Credito y cobranza. Mexico: Grupo Editorial Patria.

Orfali, C., Maman, J., \& Muñoz, A. (2017). Oracle APEX 5.1. Mexico: Clarisa Maman Orfali.

Reyno, J., Jhabvala, F., Ibanez, J., \& Martinez, R. (2000). Subdesarrollo, mercado y deuda. Mexico: Universidad Iberoamericana.

Ripia, J., Gallard, M., \& Molina, V. (1976). Practica de la administracion, y cobranza de las rentas, y visita de los ministros. Mexico: Editorial S.A.

Thibaud, C. (2006). MySQL 5: instalación, implementación, administración, programación. Barcelona: Ediciones ENI.

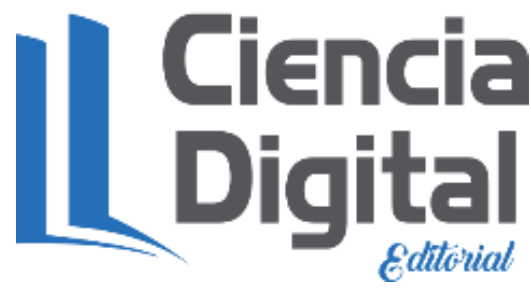




\section{Para citar el artículo indexado.}

Velasteguí E \& Sánchez C. (2017). La gestión de cobranza y la automatización por una base de datosg. Revista electrónica Visionario Digital 1(1), 19-33. Recuperado desde: http://cienciadigital.org/revistacienciadigital2/index.php/VisionarioDigital/article/view/ $\underline{257 / 560}$

\section{Liencia}

El artículo que se publica es de exclusiva responsabilidad de los autores y no necesariamente reflejan el pensamiento de la Revista Ciencia Digital.

El articulo queda en propiedad de la revista y, por tanto, su publicación parcial y/o total en otro medio tiene que ser autorizado por el director de la Revista Ciencia Digital 